\title{
CONTINUOUS DEPENDENCE FOR SOLUTION CLASSES OF EULER-LAGRANGE EQUATIONS GENERATED BY LINEAR GROWTH ENERGIES
}

\author{
KEN SHIRAKAWA \\ Department of Applied Mathematics, Graduate School of Engineering, Kobe University \\ 1-1 Rokkodai, Nada, Kobe, 657-8501, Japan \\ E-mail: sirakawa@cs.kobe-u.ac.jp
}

\begin{abstract}
In this paper, a one-dimensional Euler-Lagrange equation associated with the total variation energy, and Euler-Lagrange equations generated by approximating total variations with linear growth, are considered. Each of the problems presented can be regarded as a governing equation for steady-states in solid-liquid phase transitions. On the basis of precise structural analysis for the solutions, the continuous dependence between the solution classes of approximating problems and that of the limiting Euler-Lagrange equation will be studied by means of the analytical methods of set-valued analysis.
\end{abstract}

1. Introduction. Let $\Omega$ be the one-dimensional open interval $(0,1)$, let $\kappa>0$ be a small and fixed constant, and let $-1<\theta_{*}<1$ be a given constant.

In this paper, the following inclusions:

$$
\begin{gathered}
-\kappa\left(\frac{D w}{|D w|}\right)_{x}+\partial I_{[-1,1]}(w) \ni w+\theta_{*} \text { in } \Omega, \\
-\kappa\left(\frac{D w}{\sqrt{\varepsilon^{2}+|D w|^{2}}}\right)_{x}+\partial I_{[-1,1]}(w) \ni w+\theta_{*} \text { in } \Omega \text { for } 0<\varepsilon<1,
\end{gathered}
$$

subject to homogeneous Neumann type boundary conditions, are considered, where $\partial I_{[-1,1]}$ is the subdifferential of the indicator function $I_{[-1,1]}$ on the closed interval $[-1,1]$.

The first inclusion (1) is derived as the Euler-Lagrange equation of the following functional:

$$
w \in L^{2}(\Omega) \mapsto \mathcal{F}_{0}(w):=\kappa \operatorname{Var}(w ; \Omega)+\int_{\Omega}\left(I_{[-1,1]}(w)-\frac{1}{2}\left|w+\theta_{*}\right|^{2}\right) d x,
$$

2000 Mathematics Subject Classification: Primary 35J20; Secondary 58C06.

Key words and phrases: Euler-Lagrange equations, structural analysis, continuous dependence of solution classes.

The paper is in final form and no version of it will be published elsewhere. 
which includes the total variation $\operatorname{Var}(w ; \Omega)\left(=\int_{\Omega}|D w|\right)$ of the parameter $w \in L^{2}(\Omega)$. So, the inclusion (1) is just formal, and the rigorous one is

$$
(P)_{0} \quad \kappa \partial V_{0}(w) \ni w+\theta_{*} \text { in } L^{2}(\Omega),
$$

with the use of the $L^{2}$-subdifferential $\partial V_{0}$ of the following proper l.s.c. and convex function:

$$
z \in L^{2}(\Omega) \mapsto V_{0}(z):=\operatorname{Var}(z ; \Omega)+\int_{\Omega} I_{[-1,1]}(z) d x
$$

or equivalently, in the form of the variational inequality:

$$
\kappa V_{0}(w)-\int_{\Omega}\left(w+\theta_{*}\right) w d x \leq \kappa V_{0}(z)-\int_{\Omega}\left(w+\theta_{*}\right) z d x \text { for any } z \in D_{*},
$$

with the use of the domain:

$$
D_{*}:=\{\zeta \in B V(\Omega)|| \zeta \mid \leq 1, \text { a.e. in } \Omega\}
$$

of test functions. Here, let us note that the above $D_{*}$ coincides with the effective domain $D\left(V_{0}\right)$ of the convex function $V_{0}$.

The functional $\mathcal{F}_{0}$, given in $(3)$, is an energy functional, which was proposed and studied by Visintin [7, Chapter VI] as a possible choice of the free energy in solid-liquid phase transitions. In the context, $w=w(x)$ denotes the nonconserved order parameter, and $\theta_{*}$ denotes the (constant) relative temperature. Also, in (3), the part of the total variation is called interfacial energy, and this term is supposed to contribute the pattern formations of the interfaces. On the other hand, another integral part is called bulk energy, and this term is supposed to characterize the bi-stability of solid-liquid phases by the double-well function:

$$
\omega \in \boldsymbol{R} \mapsto I_{[-1,1]}(\omega)-\frac{1}{2}\left|\omega+\theta_{*}\right|^{2}
$$

as in the integral. In view of this, the problem $(P)_{0}$ (or inclusion (1)) can be regarded as a governing equation of the steady-states in solid-liquid phase transitions, under the constant equilibrium temperature.

For any $0<\varepsilon<1$, the second inclusion (2) is derived as the Euler-Lagrange equation for the following free energy:

$$
w \in L^{2}(\Omega) \mapsto \kappa \operatorname{Var}_{\varepsilon}(w ; \Omega)+\int_{\Omega}\left\{I_{[-1,1]}(w)-\frac{1}{2}\left|w+\theta_{*}\right|^{2}\right\} d x
$$

which includes the approximating total variation:

$$
z \in L^{1}(\Omega) \mapsto \operatorname{Var}_{\varepsilon}(z ; \Omega):=\inf \left\{\begin{array}{l|l}
\underset{\lim }{i \rightarrow+\infty} \int_{\Omega} \sqrt{\varepsilon^{2}+\left(\varphi_{i}\right)_{x}^{2}} d x & \begin{array}{l}
\left\{\varphi_{i}\right\} \subset C^{1}(\Omega), \\
\varphi_{i} \rightarrow z \operatorname{in} L^{1}(\Omega) \\
\text { as } i \rightarrow+\infty
\end{array}
\end{array}\right\}
$$

with linear growth. So, for any $0<\varepsilon<1$, the formal expression (2) is rigorously provided in the form of:

$$
(P)_{\varepsilon} \quad \partial V_{\varepsilon}(w) \ni w+\theta_{*} \text { in } L^{2}(\Omega),
$$

with the use of the $L^{2}$-subdifferential $\partial V_{\varepsilon}$ of the following proper l.s.c. and convex function on $L^{2}(\Omega)$ :

$$
z \in L^{2}(\Omega) \mapsto V_{\varepsilon}(z):=\operatorname{Var}_{\varepsilon}(z ; \Omega)+\int_{\Omega} I_{[-1,1]}(z) d x
$$


or equivalently, in the form of the variational inequality:

$$
\kappa V_{\varepsilon}(w)-\int_{\Omega}\left(w+\theta_{*}\right) w d x \leq \kappa V_{\varepsilon}(z)-\int_{\Omega}\left(w+\theta_{*}\right) z d x \text { for any } z \in D_{*},
$$

with the use of the same domain $D_{*}$ as in (4). Here, let us note that $D_{*}$ coincides with effective domains of convex functions $V_{\varepsilon}$, for all $0<\varepsilon<1$.

Now, for the sequence $\left\{V_{\varepsilon} \mid 0<\varepsilon<1\right\}$ of convex functions, it is easily checked that $V_{\varepsilon}$ converges to $V_{0}$ in the sense of Mosco [4] as $\varepsilon \searrow 0$. Hence, the problem $(P)_{0}$ can be regarded as a limiting problem of problems $(P)_{\varepsilon}$ as $\varepsilon \searrow 0$, and the approximating problems $(P)_{\varepsilon}(0<\varepsilon<1)$ are supposed to represent some regularized situations for the solutions of the problem $(P)_{0}$.

Recently, the structural analysis for solutions of $(P)_{0}$ has been studied in [3], and the reported results have enabled us to see concrete patterns of steady-states (steady-state patterns) represented by $(P)_{0}$. Therefore, applying a similar approach to approximating problems $(P)_{\varepsilon}(0<\varepsilon<1)$, we can expect to know the precise correspondence between the steady-states represented by the limiting problem $(P)_{0}$, and those represented by the approximating problems $(P)_{\varepsilon}(0<\varepsilon<1)$.

Our main focus will be on:

- structural analysis for solutions of approximating problems $(P)_{\varepsilon}(0<\varepsilon<1)$;

- limiting properties for the solution classes of $(P)_{\varepsilon}$ as $\varepsilon \searrow 0$, and comparison with the solution class of $(P)_{0}$.

Consequently, the above approximating situations will be exactly demonstrated, and the $\varepsilon$-convergence of solution classes will be characterized by means of analytical methods of set-valued analysis.

2. Preliminaries. Throughout this paper, the class of all Borel subsets in $\Omega(=(0,1))$ is denoted by $\mathcal{B}(\Omega)$, and the measure theoretical notations, such as "a.e.", " $d x$ ", and so on, are all with respect to the one-dimensional Lebesgue measure $\mathcal{L}^{1}$, if not otherwise specified.

For an abstract Banach space $X$, we denote by $|\cdot|_{X}$ the norm of $X$, and denote by $\operatorname{dist}_{X}(\xi, A)$ the distance between a point $\xi \in X$ and a subset $A \subset X$, defined as $\operatorname{dist}_{X}(\xi, A):=\inf _{\alpha \in A}|\xi-\alpha|_{X}$.

For any $L^{1}(\Omega)$, we denote by $|D z|$ the so-called total variation measure of $z$, and we call $z$ a function of bounded variation, or simply BV-function, if:

$$
|D z|(\Omega)=\operatorname{Var}(z ; \Omega):=\sup \left\{\begin{array}{l|l}
\int_{\Omega} z \varphi_{x} d x & \begin{array}{l}
\varphi \in C_{\mathrm{c}}^{1}(\Omega) \text { and } \\
|\varphi| \leq 1 \text { on } \Omega
\end{array}
\end{array}\right\}<+\infty .
$$

The class of all BV-functions is denoted by $B V(\Omega)$, and then the functional space $B V(\Omega)$ forms a Banach space endowed with the norm:

$$
|z|_{B V(\Omega)}:=|z|_{L^{1}(\Omega)}+\operatorname{Var}(z ; \Omega) \text { for any } z \in B V(\Omega) .
$$

Also, the space $B V(\Omega)$ is continuously embedded into $L^{\infty}(\Omega)$ and compactly embedded into $L^{1}(\Omega)$. Additionally, with regard to one-dimensional BV-functions, the following properties are fundamental (cf. [1, Chapter 3]). 
(BV1) For any $z \in B V(\Omega)$, there exists a fine representation $\bar{z}: \bar{\Omega} \rightarrow \boldsymbol{R}$, such that:

(i) $\bar{z}$ is described by a difference of two monotone functions, hence $\bar{z}$ admits the right-hand limits $\bar{z}(x+)$ at all $x \in[0,1)$, and the left-hand limits $\bar{z}(x-)$ at all $x \in(0,1]$;

(ii) $\bar{z}(0)=\bar{z}(0+), \bar{z}(1)=\bar{z}(1-)$, and $\bar{z}$ is continuous except on an at most countable set $A_{z}:=\{y \in \Omega|| D z \mid(\{y\}) \neq 0\}$

(iii) $\bar{z}$ is differentiable except on a zero-set $S_{z}$ with respect to $\mathcal{L}^{1}$, hence the derivative $\bar{z}_{x}(x)$ (approximate differential) exists for all $x \in \Omega \backslash S_{z}$;

(iv) the total variation $\operatorname{Var}(z ; \Omega)$ of $z$ coincides with the following quantity

$$
\operatorname{pVar}(\bar{z} ; \bar{\Omega}):=\sup \left\{\begin{array}{l|l}
\sum_{i=1}^{n}\left|\bar{z}\left(y_{i}\right)-\bar{z}\left(y_{i-1}\right)\right| & \begin{array}{l}
n \in \boldsymbol{N} \text { and } \\
0=y_{0}<y_{1}<\cdots<y_{n}=1
\end{array}
\end{array}\right\},
$$

called the pointwise variation of $\bar{z}$.

(BV2) For the total variation measure $|D z|$ of any $z \in B V(\Omega)$, let us denote by $\left|D z^{a}\right|$ and $\left|D z^{s}\right|$, respectively, the absolutely continuous part and the singular part of $|D z|$, with respect to $\mathcal{L}^{1}$. Then:

$$
\left|D z^{a}\right|(B)=\int_{B}\left|\bar{z}_{x}\right| d x \text { and }\left|D z^{s}\right|(B)=\sum_{x \in A_{z} \cap B}|\bar{z}(x+)-\bar{z}(x-)|+\left|D z^{c}\right|(B)
$$

for any $B \in \mathcal{B}(\Omega)$, where $\left|D z^{c}\right|$ is the so-called Cantor part of $|D z|$, defined as:

$$
\left|D z^{c}\right|(B):=\left|D z^{s}\right|\left(B \cap\left(S_{z} \backslash A_{z}\right)\right) \text { for any } B \in \mathcal{B}(\Omega) \text {. }
$$

Now, a BV-function $z$ is called a function of special bounded variation, or simply SBV-function, if $\left|D z^{c}\right| \equiv 0$ in $\mathcal{B}(\Omega)$.

On the basis of the above properties, the representation of the approximating total variation $\operatorname{Var}_{\varepsilon}(z ; \Omega)$, for every $0<\varepsilon<1$ and $z \in L^{1}(\Omega)$, is characterized as follows (cf. [1, Chapter 5]).

(BV3) For any $0<\varepsilon<1$, the functional $z \in L^{1}(\Omega) \mapsto \operatorname{Var}_{\varepsilon}(z ; \Omega)$ is proper, l.s.c. and convex in $L^{1}(\Omega)$, and the effective domain of this convex functional coincides with the space $B V(\Omega)$.

(BV4) For any $z \in B V(\Omega)$, there exists a unique Radon measure $\sqrt{\varepsilon^{2}+|D z|^{2}}$ such that $\operatorname{Var}_{\varepsilon}(z ; \Omega)=\sqrt{\varepsilon^{2}+|D z|^{2}}(\Omega)$, and

$$
\int_{B} \sqrt{\varepsilon^{2}+|D z|^{2}}=\int_{B} \sqrt{\varepsilon^{2}+\bar{z}_{x}^{2}} d x+\int_{B}\left|D z^{s}\right| \text { for any } B \in \mathcal{B}(\Omega) .
$$

REMARK 1. In general, BV-functions can be defined on any open interval in a manner similar to (7). Then, analogous properties to (BV1)-(BV4) are also observed for any BVfunction defined on any open interval. Especially, in the light of (BV1), we subsequently identify any BV-function with its fine representation.

Next, for arbitrary $0<\varepsilon<1$ and arbitrary constants $a, b \in \boldsymbol{R}$, let us denote by $(C P ; a, b)_{\varepsilon}$ the following Cauchy problem for a second order ordinary differential equation: 


$$
(C P ; a, b)_{\varepsilon} \quad\left\{\begin{array}{l}
-\kappa\left(\frac{\gamma_{x}(x)}{\sqrt{\varepsilon^{2}+\gamma_{x}(x)^{2}}}\right)_{x}=\gamma(x)+\theta_{*}, \quad x \in \boldsymbol{R} \\
\gamma(0)=a, \gamma_{x}(0)=b
\end{array}\right.
$$

which will be an important key in the structural analysis for solutions of approximating problems. With regard to this Cauchy problem, it is not so difficult to see the following properties.

Proposition 1 (Key properties for $\left.(C P ; a, b)_{\varepsilon}\right)$. Let us fix any $0<\varepsilon<1$. Then, the following three statements hold.

(I) For all $a, b \in \boldsymbol{R}$, there exists a certain open interval $I^{(a, b)} \subset \boldsymbol{R}$ such that $0 \in I^{(a, b)}$ and the Cauchy problem $(C P ; a, b)_{\varepsilon}$ admits a unique solution $\gamma \in C^{2}\left(I^{(a, b)}\right)$ on $I^{(a, b)}$. Furthermore, if $\gamma+\theta_{*}>0\left(\right.$ resp. $\left.\gamma+\theta_{*}<0\right)$ in $I^{(a, b)}$, then the solution $\gamma$ is strictly concave (resp. strictly convex) in $I^{(a, b)}$.

(II) If $a+\theta_{*}=0$ and $b \neq 0$, then there exists a unique constant $\lambda_{\varepsilon}^{(b)}>0$ fulfilling:

(i) $\lambda_{\varepsilon}^{(b)}>\sqrt{\kappa /(2 \varepsilon)}$ and the open interval $\left(-\lambda_{\varepsilon}^{(b)}, \lambda_{\varepsilon}^{(b)}\right)$ can be taken as the existence domain $I^{\left(-\theta_{*}, b\right)}$ as in the assertion $(I)$;

(ii) for the solution $\gamma$ of $\left(C P ;-\theta_{*}, b\right)_{\varepsilon}$, the translation $\gamma+\theta_{*}$ is an odd function on $\left(-\lambda_{\varepsilon}^{(b)}, \lambda_{\varepsilon}^{(b)}\right), \quad\left|\gamma\left(\lambda_{\varepsilon}^{(b)}-\right)+\theta_{*}\right|=\left|\gamma\left(-\lambda_{\varepsilon}^{(b)}+\right)+\theta_{*}\right|<\sqrt{2 \kappa \varepsilon}, \quad\left|\gamma_{x}\left(\lambda_{\varepsilon}^{(b)}-\right)\right|=$ $\left|\gamma_{x}\left(-\lambda_{\varepsilon}^{(b)}+\right)\right|=0$ and $\left|\gamma+\theta_{*}\right|_{L^{1}\left(0, \lambda_{\varepsilon}^{(b)}\right)}<\kappa$.

(III) If $\left|a+\theta_{*}\right| \geq \sqrt{2 \kappa \varepsilon}$ and $b=0$, then there exists a unique constant $\mu_{\varepsilon}^{(a)}>0$ fulfilling:

(iii) $\mu_{\varepsilon}^{(a)}<2 \kappa /\left|a+\theta_{*}\right|$ and the open interval $\left(-\mu_{\varepsilon}^{(a)}, \mu_{\varepsilon}^{(a)}\right)$ can be taken as the existence domain $I^{(a, 0)}$ as in the assertion $(I)$;

(iv) for the solution $\gamma$ of $(C P ; a, 0)_{\varepsilon}$, the translation $\gamma+\theta_{*}$ is an even function with no sign-change on $\left(-\mu_{\varepsilon}^{(a)}, \mu_{\varepsilon}^{(a)}\right),\left|\gamma\left(\mu_{\varepsilon}^{(a)}-\right)+\theta_{*}\right|=\left|\gamma\left(-\mu_{\varepsilon}^{(a)}+\right)+\theta_{*}\right|=\sqrt{\left|a+\theta_{*}\right|^{2}-2 \kappa \varepsilon}$, $\left|\gamma_{x}\left(\mu_{\varepsilon}^{(a)}-\right)\right|=\left|\gamma_{x}\left(-\mu_{\varepsilon}^{(a)}+\right)\right|=+\infty$, and $\left|\gamma+\theta_{*}\right|_{L^{1}\left(0, \mu_{\varepsilon}^{(a)}\right)}=\kappa$;

(v) if $0<\varepsilon_{0}<1$ and $\left|a_{0}+\theta_{*}\right|>\sqrt{2 \kappa \varepsilon_{0}}$, then

$$
\left\{\begin{array}{l}
\bullet \text { a function } a \in \boldsymbol{R} \backslash\left[-\theta_{*}-\sqrt{2 \kappa \varepsilon_{0}},-\theta_{*}+\sqrt{2 \kappa \varepsilon_{0}}\right] \mapsto \mu_{\varepsilon_{0}}^{(a)} \text { is continuous } \\
\text { and strictly decreasing }\left(\text { resp. strictly increasing) in }\left(-\theta_{*}+\sqrt{2 \kappa \varepsilon_{0}},+\infty\right)\right. \\
\text { (resp. in } \left.\left(-\infty,-\theta_{*}-\sqrt{2 \kappa \varepsilon_{0}}\right)\right) ; \\
\bullet \text { a function } \varepsilon \in\left(0, \varepsilon_{0}\right) \mapsto \mu_{\varepsilon}^{\left(a_{0}\right)} \text { is continuous and strictly increasing, } \\
\text { and } \mu_{\varepsilon}^{\left(a_{0}\right)} \searrow \kappa /\left|a_{0}+\theta_{*}\right| \text { as } \varepsilon \searrow 0 .
\end{array}\right.
$$

Proof. Assertions (I)-(III) are checked by applying the Cauchy-Lipschitz theorem to the equivalent vectorial formula to $(C P ; a, b)_{\varepsilon}(a, b \in \boldsymbol{R})$ :

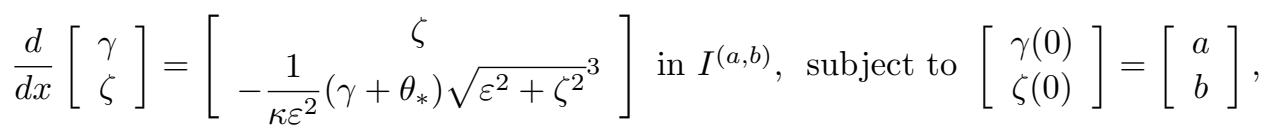


and the following equalities:

$$
\begin{gathered}
-\kappa \frac{\gamma_{x}(t)}{\sqrt{\varepsilon^{2}+\gamma_{x}(t)^{2}}}+\kappa \frac{\gamma_{x}(s)}{\sqrt{\varepsilon^{2}+\gamma_{x}(s)^{2}}}=\int_{s}^{t}\left(\gamma+\theta_{*}\right) d x, \\
\frac{\kappa \varepsilon^{2}}{\sqrt{\varepsilon^{2}+\gamma_{x}(t)^{2}}}-\frac{\kappa \varepsilon^{2}}{\sqrt{\varepsilon^{2}+\gamma_{x}(s)^{2}}}=\frac{1}{2}\left|\gamma(t)+\theta_{*}\right|^{2}-\frac{1}{2}\left|\gamma(s)+\theta_{*}\right|^{2},
\end{gathered}
$$

for all $t, s \in I^{(a, b)}$ with $t \geq s$, which are directly derived from the differential equation in $(C P ; a, b)_{\varepsilon}$. Incidentally, the item (v) of (III) is a direct consequence of the following key equality:

$$
\mu_{\varepsilon}^{(a)}=\kappa \int_{0}^{1} \frac{1-\xi}{\sqrt{\xi(2-\xi)}} \cdot \frac{1}{\sqrt{\left|a+\theta_{*}\right|^{2}-2 \kappa \varepsilon \xi}} d \xi
$$

which is obtained by using (8) under $s=0$ and standard techniques of the integration by substitution.

In the rest, we set:

$$
0<\kappa<\kappa_{*}:=\frac{1-\left|\theta_{*}\right|}{4} \text { and } 0<\varepsilon<\varepsilon_{\kappa}:=\frac{\kappa}{2},
$$

and for any $0<\varepsilon<\varepsilon_{\kappa}$, any $a \in \boldsymbol{R}$ with $\left|a+\theta_{*}\right| \geq \sqrt{2 \kappa \varepsilon}$ and any $\ell \geq \mu_{\varepsilon}^{(a)}$, we denote by $\gamma_{\varepsilon}^{(a)}$ a $C^{1}$-function on $(-\ell, \ell)$, defined as:

$$
\gamma_{\varepsilon}^{(a)}(x ; \ell):=\left\{\begin{array}{l}
\gamma\left(x-\left(\ell-\mu_{\varepsilon}^{(a)}\right)\right), \text { if } \ell-\mu_{\varepsilon}^{(a)}<x<\ell, \\
\gamma(0), \text { if }-\ell+\mu_{\varepsilon}^{(a)} \leq x \leq \ell-\mu_{\varepsilon}^{(a)}, \\
\gamma\left(x+\left(\ell-\mu_{\varepsilon}^{(a)}\right)\right), \text { if }-\ell<x<-\ell+\mu_{\varepsilon}^{(a)},
\end{array}\right.
$$

with the use of the solution $\gamma \in C^{2}\left(-\mu_{\varepsilon}^{(a)}, \mu_{\varepsilon}^{(a)}\right)$ of $(C P ; a, 0)_{\varepsilon}$.

3. Statement of the main results. Let us start by referring to the result reported in [3], which is concerned with the structure of solutions of the limiting problem $(P)_{0}$.

Proposition 2 (cf. [3, Theorem 1.1]). Let us denote by $\mathcal{S}(0)$ the class of all solutions of the problem $(P)_{0}$. Then, in the space $L^{2}(\Omega)$, the class $\mathcal{S}(0)$ coincides with the union $\bigcup_{n=0}^{+\infty} \mathcal{S}_{n}(0)$ of functional classes $\mathcal{S}_{n}(0)(n=0,1,2, \ldots)$, defined as follows.

$(S 0)_{0} \quad \mathcal{S}_{0}(0):=\left\{-1,-\theta_{*}, 1\right\}$.

$(S 1)_{0}$ For any $n \in \boldsymbol{N}, z \in \mathcal{S}_{n}(0)$ if and only if $z$ is expressed by:

$$
z(x)=\sum_{k=0}^{n}\left(c_{k}+\theta_{*}\right) \chi_{J_{k}}(x)-\theta_{*} \text { for a.e. } x \in \Omega,
$$

with constants $c_{k} \in[-1,1] \backslash\left\{-\theta_{*}\right\}$ and open intervals $J_{k}=\left(x_{k}^{R}, x_{k+1}^{L}\right)(k=$ $0,1, \ldots, n)$ determined by partition points:

$$
x_{0}^{L}=x_{0}^{R}<0<x_{1}^{L} \leq x_{1}^{R}<\cdots<x_{n}^{L} \leq x_{n}^{R}<1<x_{n+1}^{L}=x_{n+1}^{R}
$$

such that (see also Fig.1):

$(s 0)_{0} x_{0}^{L}=x_{0}^{R}=-x_{1}^{L}$ and $x_{n+1}^{L}=x_{n+1}^{R}=2-x_{n}^{R}$;

$(s 1)_{0}\left(c_{k}+\theta_{*}\right)\left(c_{k-1}+\theta_{*}\right)<0, k=1, \ldots, n$;

(s2) $)_{0}\left|c_{k}+\theta_{*}\right| \mathcal{L}^{1}\left(J_{k}\right)\left\{\begin{array}{l}\geq 2 \kappa, \text { if }\left|c_{k}\right|=1, \\ =2 \kappa, \text { if }\left|c_{k}\right|<1,\end{array} \quad k=0,1, \ldots, n\right.$. 


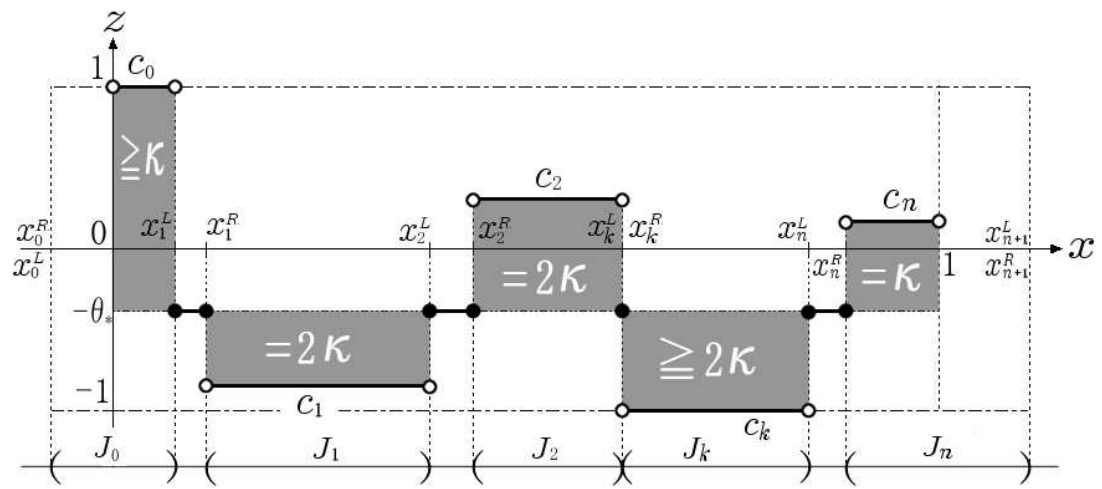

Fig. 1

REMARK 2. In the light of the conditions (9) and $(\mathrm{s} 2)_{0}$ of $(\mathrm{S} 1)_{0}$ of Proposition 2, there exists a certain maximal number $n(0) \in \boldsymbol{N}$ such that $\mathcal{S}_{n}(0) \neq \emptyset$, if $0 \leq n \leq n(0)$, and otherwise empty.

In this paper, two theorems will be stated as the main results. The first theorem is concerned with the structure of solutions of $(P)_{\varepsilon}$ for $0<\varepsilon<\varepsilon_{\kappa}$.

THEOREM 1 (Structural theorem for approximating solutions). Let us assume $0<\varepsilon<\varepsilon_{\kappa}$, and let us denote by $\mathcal{S}(\varepsilon)$ the class of all solutions of the approximating problem $(P)_{\varepsilon}$. Then, in the space $L^{2}(\Omega)$, the class $\mathcal{S}(\varepsilon)$ coincides with the union $\bigcup_{n=0}^{+\infty} \mathcal{S}_{n}(\varepsilon)$ of functional classes $\mathcal{S}_{n}(\varepsilon)(n=0,1,2, \cdots)$ defined as follows.

$(S 0)_{\varepsilon} \mathcal{S}_{0}(\varepsilon):=\left\{-1,-\theta_{*}, 1\right\}$.

$(S 1)_{\varepsilon}$ For any $n \in \boldsymbol{N}, z \in \mathcal{S}_{n}(\varepsilon)$ if and only if $z$ is expressed by:

$$
z(x)=\sum_{k=0}^{n}\left(\gamma_{\varepsilon, k}(x)+\theta_{*}\right) \chi_{J_{\varepsilon, k}}(x)-\theta_{*} \text { for a.e. } x \in \Omega,
$$

with functions $\gamma_{\varepsilon, k} \in B V(\Omega)$, open intervals $J_{\varepsilon, k}:=\left(x_{\varepsilon, k}, x_{\varepsilon, k+1}\right)(k=0,1, \ldots, n)$ determined by partition points:

$$
x_{\varepsilon, 0}<0<x_{\varepsilon, 1}<\cdots<x_{\varepsilon, n}<1<x_{\varepsilon, n+1}
$$

such that (see also Fig. 2):

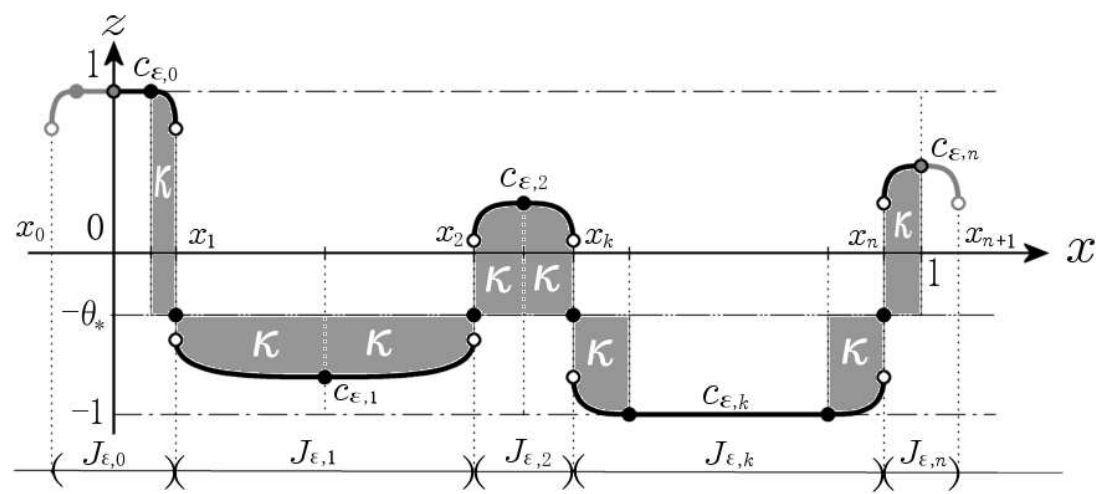

Fig. 2 
$(s 0)_{\varepsilon} x_{\varepsilon, 0}=-x_{\varepsilon, 1}$ and $x_{\varepsilon, n+1}=2-x_{\varepsilon, n}$;

$(s 1)_{\varepsilon}$ for every $k=0,1, \ldots, n, \gamma_{\varepsilon, k} \equiv-\theta_{*}$ on $\Omega \backslash J_{\varepsilon, k}$, and there exists a unique triplet $\left[\bar{x}_{\varepsilon, k}, \ell_{\varepsilon, k}, c_{\varepsilon, k}\right]$ of a spatial point $\bar{x}_{\varepsilon, k} \in \bar{\Omega}$ and constants $\ell_{\varepsilon, k}>0$ and $c_{\varepsilon, k} \in[-1,1] \backslash\left\{-\theta_{*}\right\}$, satisfying

$$
\left\{\begin{array}{l}
\bar{x}_{\varepsilon, k}=\frac{x_{\varepsilon, k+1}+x_{\varepsilon, k}}{2}, \ell_{\varepsilon, k}=\frac{x_{\varepsilon, k+1}-x_{\varepsilon, k}}{2} \\
\gamma_{\varepsilon, k}(x)=\gamma_{\varepsilon}^{\left(c_{\varepsilon, k}\right)}\left(x-\bar{x}_{\varepsilon, k} ; \ell_{\varepsilon, k}\right) \text { for any } x \in J_{\varepsilon, k},
\end{array}\right.
$$

where $\gamma_{\varepsilon}^{(\cdot)}(\cdot ; \cdot)$ is the $C^{1}$-function given in (10);

(s2) $)_{\varepsilon}\left(c_{\varepsilon, k}+\theta_{*}\right)\left(c_{\varepsilon, k-1}+\theta_{*}\right)<0, \quad k=1, \ldots, n$;

(s3) $\varepsilon_{\varepsilon}\left|\gamma_{\varepsilon, k}+\theta_{*}\right|_{L^{1}\left(J_{\varepsilon, k}\right)}\left\{\begin{array}{l}\geq 2 \kappa, \text { if }\left|c_{\varepsilon, k}\right|=1, \\ =2 \kappa, \text { if }\left|c_{\varepsilon, k}\right|<1,\end{array} \quad k=0,1, \ldots, n\right.$.

REMARK 3. A similar situation as in Remark 2 is also observed in the above Theorem 1. Namely, when $0<\varepsilon<\varepsilon_{\kappa}$, the properties reported in (III) of Proposition 1 imply the existence of the maximal number $n(\varepsilon) \in \boldsymbol{N}$, such that $\mathcal{S}_{n}(\varepsilon) \neq \emptyset$, if $0 \leq n \leq n(\varepsilon)$, and otherwise empty. Especially, from (v) of (III) of Proposition 1, we further see $n(\varepsilon) \leq n(0)$, where $n(0)$ is the maximal number mentioned in Remark 2.

On account of Proposition 2 and Theorem 1, we can naturally identify the notation $\mathcal{S}(\varepsilon)\left(0 \leq \varepsilon<\varepsilon_{\kappa}\right)$ with the following set-valued mapping:

$$
\varepsilon \in\left[0, \varepsilon_{\kappa}\right) \mapsto \mathcal{S}(\varepsilon) \subset X_{*}:=\left\{z \in D_{*}|| D z \mid(\Omega) \leq 2 n(0)\right\}
$$

from the interval $\left[0, \varepsilon_{\kappa}\right)$ into the compact subset $X_{*}$ in $L^{2}(\Omega)$.

Now, the second theorem is concerned with the limiting observation of the above set-valued mapping $\mathcal{S}=\mathcal{S}(\varepsilon)$ as $\varepsilon \searrow 0$.

TheOREM 2 (Limiting observation as $\varepsilon \searrow 0$ ). For the set-valued mapping, given in (12), the limit-supremum:

$$
\varlimsup_{\varepsilon \searrow 0} \mathcal{S}(\varepsilon):=\left\{z \in L^{2}(\Omega) \mid \underline{\lim }_{\varepsilon \searrow 0} \operatorname{dist}_{L^{2}(\Omega)}(z, \mathcal{S}(\varepsilon))=0\right\}
$$

and the limit-infimum:

$$
\underline{\lim }_{\varepsilon \searrow 0} \mathcal{S}(\varepsilon):=\left\{z \in L^{2}(\Omega) \mid \lim _{\varepsilon \searrow 0} \operatorname{dist}_{L^{2}(\Omega)}(z, \mathcal{S}(\varepsilon))=0\right\} ;
$$

are nonempty and coincide with each other, namely the limit $\mathcal{S}_{*}(0):=\lim _{\varepsilon \backslash 0} \mathcal{S}(\varepsilon)$ exists. Moreover, this limit is the union $\bigcup_{n=0}^{n(0)} \mathcal{S}_{*, n}(0)$ of functional classes $\mathcal{S}_{*, n}(0)$ ( $n=$ $0,1, \ldots, n(0))$, defined as $\mathcal{S}_{*, 0}(0):=\left\{-1,-\theta_{*}, 1\right\}$, and:

$$
\mathcal{S}_{*, n}(0):=\left\{\begin{array}{l|l}
z \in \mathcal{S}_{n}(0) & \begin{array}{l}
\circ x_{k}^{L}=x_{k}^{R} \text { for } k=1, \ldots, n \\
\circ\left|c_{k_{0}}\right|<1 \text { or }\left|c_{k_{0}}+\theta_{*}\right| \mathcal{L}^{1}\left(J_{k_{0}}\right)>2 \kappa \\
\text { for some } 0 \leq k_{0} \leq n .
\end{array}
\end{array}\right\}
$$

for $n=1, \ldots, n(0)$.

REMARK 4. As another consequence of Theorem 2 , we see that $\lim _{\varepsilon}{ }_{0} \mathcal{S}(\varepsilon) \varsubsetneqq \mathcal{S}(0)$. Therefore, by virtue of (12) and [2, Section 1.4], it is possible to conclude that the setvalued mapping $\mathcal{S}=\mathcal{S}(\varepsilon)$ is upper semi-continuous, but not lower semi-continuous, at $\varepsilon=0$. 
REMARK 5. In the case that the singular diffusion as in (1) is regularized by $p$-Laplacians, a similar conclusion as in Theorem 2 was reported in [5, Theorem 4.1]. According to the result, any approximation limit by $p$-Laplacians is required to satisfy the symmetry with respect to $-\theta_{*}$, besides conditions as in (13). In view of this, the approximation range in this paper is absolutely wider than that by $p$-Laplacians, although either approximation method can cover only restricted part of the solutions of the limiting problem $(P)_{0}$.

4. Proof of Theorem 1. We immediately check that $w$ is a constant solution of $(P)_{\varepsilon}$ if and only if $w \in \mathcal{S}_{0}(\varepsilon)$, by the method adopted in [6, Proposition 4.1]. In view of this, we deal with only nonconstant functions.

The assertion is reduced to showing that $\mathcal{S}(\varepsilon) \backslash \mathcal{S}_{0}(\varepsilon)=\bigcup_{n=1}^{n(\varepsilon)} \mathcal{S}_{n}(\varepsilon)$. We divide the argument into two subsections, to prove inclusions $\mathcal{S}(\varepsilon) \backslash \mathcal{S}_{0}(\varepsilon) \subset \bigcup_{n=1}^{n(\varepsilon)} \mathcal{S}_{n}(\varepsilon)$ and $\mathcal{S}(\varepsilon) \backslash$ $\mathcal{S}_{0}(\varepsilon) \supset \bigcup_{n=1}^{n(\varepsilon)} \mathcal{S}_{n}(\varepsilon)$, respectively.

4.1. Proof of $\mathcal{S}(\varepsilon) \backslash \mathcal{S}_{0}(\varepsilon) \subset \bigcup_{n=1}^{n(\varepsilon)} \mathcal{S}_{n}(\varepsilon)$. Let us fix arbitrary $0<\varepsilon<\varepsilon_{\kappa}$, and take any nonconstant solution $w \in \mathcal{S}(\varepsilon) \backslash \mathcal{S}_{0}(\varepsilon)$ of the problem $(P)_{\varepsilon}$. Then, the claim in this subsection is proved through some steps, stated in the following lemmas and remarks.

LEMMA 1 (Step1). Let us set:

$$
K_{\varepsilon}:=\left\{\begin{array}{l|l}
x \in \bar{\Omega} & \begin{array}{l}
\circ\left(w(x+)+\theta_{*}\right)\left(w(x-)+\theta_{*}\right) \leq 0, \text { if } x \in \Omega, \\
\circ w(x+)+\theta_{*}=0\left(\text { resp. } w(x-)+\theta_{*}=0\right), \\
\text { if } x=0 \in \partial \Omega(\text { resp. } x=1 \in \partial \Omega)
\end{array}
\end{array}\right\} .
$$

Then, $K_{\varepsilon}$ is closed subset in $\bar{\Omega}$. Hence, $\bar{\Omega} \backslash K_{\varepsilon}$ is a disjoint union of at most countably many relatively open intervals $I_{\varepsilon, k}(k=0,1,2, \ldots)$ in $\bar{\Omega}$.

Lemma 2 (Step 2). For every $k=0,1,2, \ldots, w+\theta_{*}$ has no sign-change in $I_{\varepsilon, k}$, namely $w+\theta_{*}>0$ in $I_{\varepsilon, k}$ or $w+\theta_{*}<0$ in $I_{\varepsilon, k}$.

Lemma 3 (Step 3 ). For every $k=0,1,2, \ldots$ :

(step 3-1) $w$ is continuous in $I_{\varepsilon, k}$;

(step 3-2) $w$ is concave (resp. convex) in $I_{\varepsilon, k}$, if $w+\theta_{*}>0\left(\right.$ resp. $\left.w+\theta_{*}<0\right)$ in $I_{\varepsilon, k}$.

REMARK 6. The above Lemma 3 implies that $w$ is a locally Lipschitz function (SBVfunction) on the interior $\operatorname{int}\left(I_{\varepsilon, k}\right)$ of $I_{\varepsilon, k}$ for all $k=0,1,2, \ldots$.

Lemma 4 (Step 4). For every $k=0,1,2, \ldots$, if $|w|<1$ in an open interval $I \subset \subset \operatorname{int}\left(I_{\varepsilon, k}\right)$, then $w \in C^{2}(\bar{I})$ and $w$ solves the following second order ordinary differential equation:

$$
-\kappa\left(f_{\varepsilon}\left(w_{x}\right)\right)_{x}=w+\theta_{*} ;
$$

in $I$, where $f_{\varepsilon}: \boldsymbol{R} \rightarrow(-1,1)$ is a smooth and strictly monotone function, defined as:

$$
f_{\varepsilon}(\omega):=\frac{\omega}{\sqrt{\varepsilon^{2}+\omega^{2}}} \text { for all } \omega \in \boldsymbol{R} .
$$

Lemma 5 (Step 5). Let us set $a_{\varepsilon, k}:=\inf I_{\varepsilon, k}$ and $b_{\varepsilon, k}:=\sup I_{\varepsilon, k}$, for $k=0,1,2, \ldots$ Then, for every $k=0,1,2, \ldots$ :

(step 5-1) $\left|w\left(a_{\varepsilon, k}+\right)\right|<1$ (resp. $\left.\left|w\left(b_{\varepsilon, k}-\right)\right|<1\right)$, if $a_{\varepsilon, k} \in \Omega\left(\right.$ resp. $\left.b_{\varepsilon, k} \in \Omega\right)$;

(step 5-2) $\left|w_{x}\left(a_{\varepsilon, k}+\right)\right|=+\infty$ (resp. $\left.\left|w_{x}\left(b_{\varepsilon, k}-\right)\right|=+\infty\right)$, if $a_{\varepsilon, k} \in \Omega$ (resp. $\left.b_{\varepsilon, k} \in \Omega\right)$; 
(step 5-3) $w_{x}\left(a_{\varepsilon, k}+\right)=0$ (resp. $\left.w_{x}\left(b_{\varepsilon, k}-\right)=0\right)$, if $a_{\varepsilon, k}=0 \in \partial \Omega\left(\right.$ resp. $\left.b_{\varepsilon, k}=1 \in \partial \Omega\right)$; (step 5-4) $w \in C^{1}\left(I_{\varepsilon, k}\right)$ and there exists a point $\xi_{\varepsilon, k} \in I_{\varepsilon, k}$ such that $w_{x}\left(\xi_{\varepsilon, k}\right)=0$.

REMARK 7. In view of (III) of Proposition 1 and Lemmas 4-5, it will be seen that:

$$
\mathcal{L}^{1}\left(I_{\varepsilon, k}\right) \geq \min \left\{\mu_{\varepsilon}^{(1)}, \mu_{\varepsilon}^{(-1)}\right\} \geq \frac{\kappa}{1+\left|\theta_{*}\right|} ;
$$

and hence the closed (compact) set $K_{\varepsilon} \subset \bar{\Omega}$ will be expressed as a disjoint union of at most a finite number of compact intervals. Furthermore, for any index $k$,

(7a) $w\left(a_{\varepsilon, k}+\right)+\theta_{*} \neq 0$ or $\left|w_{x}\left(a_{\varepsilon, k}+\right)\right|=+\infty$;

(7b) $w\left(b_{\varepsilon, k}-\right)+\theta_{*} \neq 0$ or $\left|w_{x}\left(b_{\varepsilon, k}-\right)\right|=+\infty$.

In fact, if one of $(7 \mathrm{a})$ and $(7 \mathrm{~b})$ does not hold, then we find a certain index $k_{1}$ and a finite constant $b_{1} \in \boldsymbol{R}$ such that $b_{1}=w_{x}\left(a_{\varepsilon, k_{1}}+\right)$ or $b_{1}=w_{x}\left(b_{\varepsilon, k_{1}}-\right)$. In either event, the following contradictory inequality:

$$
\mathcal{L}^{1}\left(I_{\varepsilon, k_{1}}\right) \geq \lambda_{\varepsilon}^{\left(b_{1}\right)}>\sqrt{\frac{\kappa}{2 \varepsilon}}>\sqrt{\frac{\kappa}{2 \varepsilon_{\kappa}}}=\sqrt{\frac{\kappa}{2} \cdot \frac{2}{\kappa}}=1=\mathcal{L}^{1}(\Omega) .
$$

will be derived from (9), (II) of Proposition 1 and (step 5-4) of Lemma 5.

Lemma 6 (Step 6$). K_{\varepsilon}$ is a finite set, and $K_{\varepsilon} \cap \partial \Omega=\emptyset$.

REMARK 8. On account of Preposition 1, Lemmas 4-6 and Remark 7, the setting of these intervals can be arranged as:

$$
I_{\varepsilon, k}:=\left\{\begin{array}{l}
{\left[a_{\varepsilon, 0}, b_{\varepsilon, 0}\right)\left(=\left[0, a_{\varepsilon, 1}\right)\right), \text { if } k=0,} \\
\left(a_{\varepsilon, k}, b_{\varepsilon, k}\right)\left(=\left(b_{\varepsilon, k-1}, a_{\varepsilon, k+1}\right)\right) \text { if } 0<k<n, \\
\left(a_{\varepsilon, n}, b_{\varepsilon, n}\right]\left(=\left(b_{\varepsilon, n-1}, 1\right]\right), \text { if } k=n,
\end{array}\right.
$$

by using a certain finite number $n \in N$ with $n \leq n(\varepsilon)$. Furthermore, putting:

$$
c_{\varepsilon, k}:=w\left(\xi_{\varepsilon, k}\right) \text { for } k=0,1, \ldots, n,
$$

Lemmas 4-5 enable us to conclude that:

$$
w(x)=\left\{\begin{array}{l}
\gamma_{\varepsilon}^{\left(c_{\varepsilon, 0}\right)}\left(x ; b_{\varepsilon, 0}\right), \text { if } x \in I_{\varepsilon, 0}, \\
\gamma_{\varepsilon}^{\left(c_{\varepsilon, k}\right)}\left(x-\frac{b_{\varepsilon, k}+a_{\varepsilon, k}}{2} ; \frac{b_{\varepsilon, k}-a_{\varepsilon, k}}{2}\right), \text { if } x \in I_{\varepsilon, k} \text { and } 0<k<n, \\
\gamma_{\varepsilon}^{\left(c_{\varepsilon, n}\right)}\left(x-1 ; 1-a_{\varepsilon, n}\right), \text { if } x \in I_{\varepsilon, n} .
\end{array}\right.
$$

LEMma 7 (Step 7). Under the reorganized settings as in (16)-(17), $\left(c_{\varepsilon, k}+\theta_{*}\right)\left(c_{\varepsilon, k-1}+\right.$ $\left.\theta_{*}\right)<0$ for $k=1, \ldots, n$.

As a consequence of all of the above facts, the nonconstant solution $w$ will be supposed to belong to the class $\mathcal{S}_{n}(\varepsilon)$ for some finite number $n \in \boldsymbol{N}$ with $n \leq n(\varepsilon)$. Then, the partition points, as in (11), will be settled as:

$$
x_{\varepsilon, k}:=\left\{\begin{array}{l}
-a_{\varepsilon, 1}=-b_{\varepsilon, 0} \text { for } k=0 \\
a_{\varepsilon, k}=b_{\varepsilon, k-1} \text { for } 1 \leq k \leq n, \\
2-a_{\varepsilon, n}=2-b_{\varepsilon, n-1} \text { for } k=n+1 .
\end{array}\right.
$$

Now, the rest of this subsection will be devoted to the proofs of Lemmas 1-7. 
Proofs of Lemmas 1-2. We refer to [3], because Lemmas 1 and 2 are proved, respectively, just by applying the same demonstration techniques adopted in [3, Lemmas 2.2 and 2.3] with slight modifications.

Proof of Lemma 3. Claims (step 3-1)-(step 3-2) are proved by means of contradiction arguments. For simplicity, let us consider only the case that $w+\theta_{*}>0$ in $I_{\varepsilon, k}(k \in$ $\boldsymbol{N} \cup\{0\}$ ), because the other case is similarly obtained.

When we prove (step 3-1), we assume the existence of a discontinuity point $x_{1}^{\circ} \in I_{\varepsilon, k}$ (actually $x_{1}^{\circ} \in \operatorname{int}\left(I_{\varepsilon, k}\right)$ ) of $w$, and define a test function $w_{1}^{\circ} \in D_{*}$ for the variational inequality (6), by putting:

$$
w_{1}^{\circ}(x):=\left\{\begin{array}{c}
\min \left\{w(x)+\left(w\left(x_{1}^{\circ}+\right)-w\left(x_{1}^{\circ}-\right)\right), 1\right\}, \\
\quad \text { if } x \in I_{\varepsilon, k}, w\left(x_{1}^{\circ}+\right)>w\left(x_{1}^{\circ}-\right) \text { and } x<x_{1}^{\circ}, \\
\min \left\{w(x)-\left(w\left(x_{1}^{\circ}+\right)-w\left(x_{1}^{\circ}-\right)\right), 1\right\}, \\
\quad \text { if } x \in I_{\varepsilon, k}, w\left(x_{1}^{\circ}+\right)<w\left(x_{1}^{\circ}-\right) \text { and } x>x_{1}^{\circ}, \\
w(x), \text { otherwise. }
\end{array}\right.
$$

Then, by using (BV1)-(BV4), Remark 1, and approximation arguments based on (5), it is not difficult to check that:

$$
V_{\varepsilon}\left(w_{1}^{\circ}\right) \leq V_{\varepsilon}(w) \text { and }-\int_{\Omega}\left(w+\theta_{*}\right)\left(w_{1}^{\circ}-w\right) d x<0 .
$$

This contradicts $(6)$.

Next, to prove (step 3-2), we temporarily negate the assumption, and take a maximal open interval $\left(a_{1}^{\circ}, b_{1}^{\circ}\right) \subset I_{\varepsilon, k}(k \in N \cup\{0\})$ such that:

$$
w\left(\tau a_{1}^{\circ}+(1-\tau) b_{1}^{\circ}\right)<\tau w\left(a_{1}^{\circ}\right)+(1-\tau) w\left(b_{1}^{\circ}\right) \text { for all } 0<\tau<1,
$$

by continuity of $w$ in $I_{\varepsilon, k}$. Here, let us define a function $w_{2}^{\circ} \in D_{*}$, by putting:

$$
w_{2}^{\circ}(x):=\left\{\begin{array}{l}
\tau w\left(a_{1}^{\circ}\right)+(1-\tau) w\left(b_{1}^{\circ}\right), \\
\quad \text { if } x=\tau a_{1}^{\circ}+(1-\tau) b_{1}^{\circ} \in\left(a_{1}^{\circ}, b_{1}^{\circ}\right) \text { with } \tau=\left(b_{1}^{\circ}-x\right) /\left(b_{1}^{\circ}-a_{1}^{\circ}\right) ; \\
w(x), \text { otherwise. }
\end{array}\right.
$$

Then, noting that the 2-dimensional segment:

$$
L_{\varepsilon}^{\circ}:=\left\{\tau\left(a_{1}^{\circ}, \frac{1}{\varepsilon} w\left(a_{1}^{\circ}\right)\right)+(1-\tau)\left(b_{1}^{\circ}, \frac{1}{\varepsilon} w\left(b_{1}^{\circ}\right)\right) \mid 0 \leq \tau \leq 1\right\} \subset \boldsymbol{R}^{2}
$$

realizes the shortest route between two points $\left(a_{1}^{\circ}, w\left(a_{1}^{\circ}\right) / \varepsilon\right),\left(b_{1}^{\circ}, w\left(b_{1}^{\circ}\right) / \varepsilon\right) \in \boldsymbol{R}^{2}$, it can be calculated that:

$$
\begin{aligned}
V_{\varepsilon}\left(w_{2}^{\circ}\right)-V_{\varepsilon}(w) & =\int_{\left(a_{1}^{\circ}, b_{1}^{\circ}\right)} \sqrt{\varepsilon^{2}+\left|D w_{2}^{\circ}\right|^{2}}-\int_{\left(a_{1}^{\circ}, b_{1}^{\circ}\right)} \sqrt{\varepsilon^{2}+|D w|^{2}} \\
& =\varepsilon\left(\int_{a_{1}^{\circ}}^{b_{1}^{\circ}} \sqrt{1+\left(\frac{1}{\varepsilon} w_{2}^{\circ}\right)_{x}^{2}} d x-\int_{\left(a_{1}^{\circ}, b_{1}^{\circ}\right)} \sqrt{1+\left|D\left(\frac{1}{\varepsilon} w\right)\right|^{2}}\right) \leq 0
\end{aligned}
$$

by approximation arguments for $w$. Also, we easily have:

$$
-\int_{\Omega}\left(w+\theta_{*}\right)\left(w_{2}^{\circ}-w\right) d x<0 .
$$

Inequalities (18)-(19) contradict (6). 
Proof of Lemma 4. This lemma is concluded by applying the usual variational method. In fact, due to the Lipschitz continuity of $w$ in $I \subset \subset \operatorname{int}\left(I_{\varepsilon, k}\right)(k \in N \cup\{0\})$, we can calculate the first variation of the variational inequality (6), to obtain:

$$
-\kappa\left[f_{\varepsilon}\left(w_{x}\right)\right]^{\prime}=w+\theta_{*} \text { in } \mathcal{D}^{\prime}(I) ;
$$

where "[ []$^{\prime \prime}$ " denotes the distributional differential, and $f_{\varepsilon}$ is the smooth and strictly increasing function given in (15).

Equality (20) implies $w \in W^{2, \infty}(I)$, since $w_{x} \in L^{\infty}(I)$ and $f_{\varepsilon}$ has a smooth inverse $f_{\varepsilon}^{-1}$ on $(-1,1)$. Furthermore, combining $(20)$, the smoothness of $f_{\varepsilon}$ and the continuity of $w_{x}$ on $\bar{I}$, we conclude that $w \in C^{2}(\bar{I})$.

Proof of Lemma 5. Claims (step 5-1)-(step 5-4) are proved by means of contradiction arguments. Then, in either claim, the key to derive a contradiction is in the point that the graph of the extension:

$$
w^{\operatorname{ex}}(x):=\left\{\begin{array}{l}
w(x), \text { if } x \in \Omega=(0,1), \\
w(0+)(\operatorname{resp} . w(1-)), \text { if } x \leq 0(\text { resp. } x \geq 1),
\end{array} \text { for any } x \in \boldsymbol{R},\right.
$$

of $w$, including the vertical segmental parts by jumps, does not have $C^{1}$-regularity (roughly, the graph has an angular shape) at some point $\left(x_{2}^{\circ}, w\left(x_{2}^{\circ}\right)\right) \in \bar{\Omega} \times[-1,1]$.

Let us demonstrate the above rough sketch, by taking the proof of (step 5-1) as an analogy. To this end, it is enough to consider only the case of $w\left(b_{\varepsilon, k}-\right)=1$, since other cases are similarly proved. In this case, it is supposed that the graph of $w^{\text {ex }}$ has an angular shape at $\left(b_{\varepsilon, k}, w\left(b_{\varepsilon, k}-\right)\right) \in \Omega \times[-1,1]$. Also, due to Lemma 4 and the concavity of $w$, it is not so difficult to check that

$$
w_{x}\left(b_{\varepsilon, k}-\right) \geq 0 \text { and } w \in C^{2}\left[b_{\varepsilon, k}-\delta_{1}^{\circ}, b_{\varepsilon, k}\right] \text { for some small } \delta_{1}^{\circ}>0 \text {. }
$$

Here, for any $0<\delta<\delta_{1}^{\circ}$, let us define a function $w_{1, \delta}^{\circ}$, by putting:

$$
w_{1, \delta}^{\circ}(x):=\left\{\begin{array}{c}
\tau w\left(b_{\varepsilon, k}-\delta\right)+(1-\tau)\left(w\left(b_{\varepsilon, k}-\delta\right)-\delta\right), \\
\quad \text { if } x=\tau\left(b_{\varepsilon, k}-\delta\right)+(1-\tau) b_{\varepsilon, k} \in I_{\varepsilon, k} \text { with } \tau=\left(b_{\varepsilon, k}-x\right) / \delta, \\
w(x), \text { otherwise, for any } x \in \Omega .
\end{array}\right.
$$

Then, taking account of (BV4), (21) and the fact that $w\left(b_{\varepsilon, k}-\right)=1>-\theta_{*} \geq w\left(b_{\varepsilon, k}+\right)$, we calculate that:

$$
\begin{aligned}
V_{\varepsilon}\left(w_{1, \delta}^{\circ}\right)-V_{\varepsilon}(w) \leq & \varepsilon\left[-\frac{\delta}{\varepsilon}-\sqrt{\delta^{2}+\left(\frac{\delta}{\varepsilon} w_{x}\left(b_{\varepsilon, k}-\right)\right)^{2}}+\sqrt{\delta^{2}+\left(\frac{\delta}{\varepsilon}\right)^{2}}\right. \\
& \left.+\left(\sqrt{\delta^{2}+\left(\frac{\delta}{\varepsilon} w_{x}\left(b_{\varepsilon, k}-\right)\right)^{2}}-\int_{b_{\varepsilon, k}-\delta}^{b_{\varepsilon, k}} \sqrt{1+\left(\frac{1}{\varepsilon} w\right)_{x}^{2}} d x\right)\right] \\
\leq & -\delta\left(1+\varepsilon-\sqrt{1+\varepsilon^{2}}\right)+O\left(\delta^{2}\right)
\end{aligned}
$$

and

$$
0 \leq-\int_{\Omega}\left(w+\theta_{*}\right)\left(w_{1, \delta}^{\circ}-w\right) d x \leq 2 \int_{b_{\varepsilon, k}-\delta}^{b_{\varepsilon, k}}\left(\delta+\delta\left|w_{x}\left(b_{\varepsilon, k}-\delta_{1}^{\circ}\right)\right|\right) d x=O\left(\delta^{2}\right), \quad \text { as } \delta \searrow 0 .
$$


Hence,

$$
\begin{gathered}
\kappa V_{\varepsilon}\left(w_{1, \delta}^{\circ}\right)-\int_{\Omega}\left(w+\theta_{*}\right) w_{1, \delta}^{\circ} d x \leq \kappa V_{\varepsilon}(w)-\int_{\Omega}\left(w+\theta_{*}\right) w d x \\
-\delta\left(1+\varepsilon-\sqrt{1+\varepsilon^{2}}\right)+O\left(\delta^{2}\right), \quad \text { as } \delta \searrow 0 .
\end{gathered}
$$

This contradicts (6).

The demonstration methods of the remaining (step 5-2)-(step 5-4) are analogous to that used in the previous lemma.

Proof of Lemma 6 . In the light of Remark 7, our goal is to show that $y_{1}^{\circ}=y_{2}^{\circ} \notin \partial \Omega=$ $\{0,1\}$, for any connected component $\left[y_{1}^{\circ}, y_{2}^{\circ}\right]$ of $K_{\varepsilon}$.

Here, if $y_{1}^{\circ}=0 \in \partial \Omega$ (resp. $y_{2}^{\circ}=1 \in \partial \Omega$ ), then a contradiction is derived from the following inequalities:

$$
V_{\varepsilon}\left(w_{3}^{\circ}\right)<V_{\varepsilon}(w) \text { and }-\int_{\Omega}\left(w+\theta_{*}\right)\left(w_{3}^{\circ}-w\right) d x<0,
$$

for a test function $w_{3}^{\circ} \in D_{*}$ for $(6)$, given as:

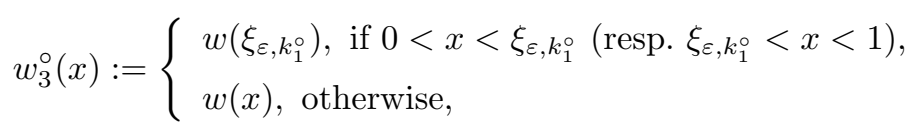

where $\xi_{\varepsilon, k_{0}^{\circ}}$ is the zero point of $w_{x}$ in $I_{\varepsilon, k_{1}^{\circ}}$, found in (step 5-4) of Lemma 5. Thus, $\left[y_{1}^{\circ}, y_{2}^{\circ}\right] \cap$ $\partial \Omega=\emptyset$, equivalently $\left[y_{1}^{\circ}, y_{2}^{\circ}\right] \subset \Omega$.

In view of this, let us next assume that $0<y_{1}^{\circ}<y_{2}^{\circ}<1$, and let us take a neighboring interval $I_{\varepsilon, k_{1}^{\circ}}$ of the interval $\left[y_{1}^{\circ}, y_{2}^{\circ}\right]$ with an index $k_{1}^{\circ} \in \boldsymbol{N} \cup\{0\}$. Then, we may say that

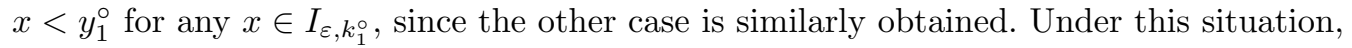
a function $w_{4}^{\circ} \in D_{*}$, defined as:

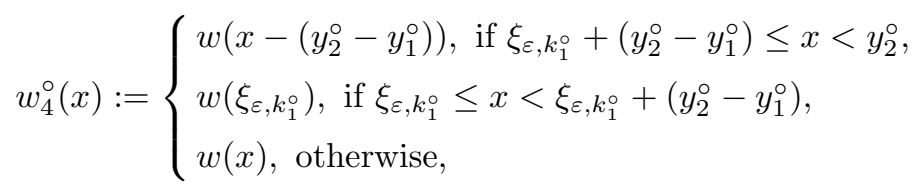

can be taken as a test function for (6), because it is actually seen that:

$$
V_{\varepsilon}\left(w_{4}^{\circ}\right) \leq V_{\varepsilon}(w) \text { and }-\int_{\Omega}\left(w+\theta_{*}\right)\left(w_{4}^{\circ}-w\right) d x<0 .
$$

Proof of Lemma 7. Under the settings as in (16)-(17), let us assume that $\left(c_{\varepsilon, k_{2}^{\circ}+}\right.$ $\left.\theta_{*}\right)\left(c_{\varepsilon, k_{2}^{\circ}-1}+\theta_{*}\right)>0$ for some $1 \leq k_{2}^{\circ} \leq n$, to show a contradiction. Then, it is enough to consider only the case that $0<c_{\varepsilon, k_{2}^{\circ}-1}+\theta_{*}<c_{\varepsilon, k_{2}^{\circ}}+\theta_{*}$, because proofs of other cases are essentially the same. In this case, we reach a contradiction with the use of a test function $w_{5}^{\circ}$ for (6), given as:

$$
w_{5}^{\circ}(x):=\left\{\begin{array}{l}
c_{k_{2}^{\circ}-1}, \text { if } \xi_{\varepsilon, k_{2}^{\circ}-1}<x<b_{\varepsilon, k_{2}^{\circ}-1}, \\
w(x), \text { otherwise }
\end{array}\right.
$$

which satisfies the following contradictory inequalities:

$$
V_{\varepsilon}\left(w_{5}^{\circ}\right)<V_{\varepsilon}(w) \text { and }-\int_{\Omega}\left(w+\theta_{*}\right)\left(w_{5}^{\circ}-w\right) d x<0 .
$$


4.2. Proof of $\mathcal{S}(\varepsilon) \backslash \mathcal{S}_{0}(\varepsilon) \supset \bigcup_{n=1}^{n(\varepsilon)} \mathcal{S}_{n}(\varepsilon)$. Let us fix any $0<\varepsilon<\varepsilon_{\kappa}$, and let us take any $w \in \bigcup_{n=1}^{n(\varepsilon)} \mathcal{S}_{n}(\varepsilon)$. Then, we may assume that $w \in \mathcal{S}_{1}(\varepsilon),-\theta_{*}<c_{\varepsilon, 0}<1, c_{\varepsilon, 1}=-1$ and $d_{\varepsilon, 1}:=\ell_{\varepsilon, 1}-\mu_{\varepsilon}^{(-1)}>0$, since the proofs for other situations are analogous. Here, let us put:

$$
\Omega_{0}:=\left(0,1-d_{\varepsilon, 1}\right) \text { and } \Omega_{1}:=\left(1-d_{\varepsilon, 1}, 1\right) .
$$

Then, taking account of (BV4), the governing ordinary differential equations (14) in both intervals $\left(0, x_{\varepsilon, 1}\right)$ and $\left(x_{\varepsilon, 1}, 1-d_{\varepsilon, 1}\right)$, and the associated boundary conditions, we calculate that:

$$
\begin{aligned}
\int_{\Omega_{0}}\left(w+\theta_{*}\right)(\varphi-w) d x=\lim _{\delta \searrow 0} \int_{\Omega_{0} \backslash\left[x_{\varepsilon, 1}-\delta, x_{\varepsilon, 1}+\delta\right]}\left(w+\theta_{*}\right)(\varphi-w) d x \\
=\kappa \lim _{\delta \backslash 0}\left(\left[-f_{\varepsilon}\left(w_{x}(x)\right)(\varphi-w)(x)\right]_{0}^{x_{\varepsilon, 1}} \bar{\tau}^{\delta}\left[-f_{\varepsilon}\left(w_{x}(x)\right)(\varphi-w)(x)\right]_{x_{\varepsilon, 1}+\delta}^{1-d_{\varepsilon, 1}}\right. \\
\left.\quad+\int_{\Omega_{0} \backslash\left[x_{\varepsilon, 1}-\delta, x_{\varepsilon, 1}+\delta\right]} f_{\varepsilon}\left(w_{x}\right)(\varphi-w)_{x} d x\right) \\
=\kappa\left(w\left(x_{\varepsilon, 1}+\right)-w\left(x_{\varepsilon, 1}-\right)\right)+\int_{\Omega_{0} \backslash\left\{x_{\varepsilon, 1}\right\}} f_{\varepsilon}\left(w_{x}\right)(\varphi-w)_{x} d x \\
\leq \kappa \sqrt{\varepsilon^{2}+|D \varphi|^{2}}\left(\Omega_{0}\right)-\kappa \sqrt{\varepsilon^{2}+|D w|^{2}}\left(\Omega_{0}\right), \text { for any } \varphi \in C^{1}\left(\Omega_{0}\right)
\end{aligned}
$$

Also, since $w \equiv-1$ in $\Omega_{1}$,

$$
\int_{\Omega_{1}}\left(w+\theta_{*}\right)(\varphi-w) d x \leq 0 \leq \kappa \sqrt{\varepsilon^{2}+|D \varphi|^{2}}\left(\Omega_{1}\right)-\kappa \sqrt{\varepsilon^{2}+|D w|^{2}}\left(\Omega_{1}\right),
$$

for any $\varphi \in C^{1}\left(\Omega_{1}\right)$. Therefore, using the standard approximation argument, and noting the fact that $w$ is a SBV-function, it is calculated that:

$$
\begin{aligned}
& \int_{\Omega}\left(w+\theta_{*}\right)(z-w) d x=\sum_{k=0}^{1} \int_{\Omega_{k}}\left(w+\theta_{*}\right)(z-w) d x \\
\leq & \sum_{k=0}^{1}\left(\kappa \sqrt{\varepsilon^{2}+|D z|^{2}}\left(\Omega_{k}\right)-\kappa \sqrt{\varepsilon^{2}+|D w|^{2}}\left(\Omega_{k}\right)\right) \leq \kappa V_{\varepsilon}(z)-\kappa V_{\varepsilon}(w),
\end{aligned}
$$

for all $z \in D_{*}$. Thus, $w$ is a nonconstant solution of $(P)_{\varepsilon}$, namely $w \in \mathcal{S}(\varepsilon) \backslash \mathcal{S}_{0}(\varepsilon)$.

5. Proof of Theorem 2. By the definitions of the limit-supremum $\overline{\lim }_{\varepsilon \backslash 0} \mathcal{S}(\varepsilon)$ and the limit-infimum $\underline{\lim }_{\varepsilon \backslash 0} \mathcal{S}(\varepsilon)$, the assertion can be restated in the form of the following inclusions:

$$
\varlimsup_{\varepsilon \searrow 0} \mathcal{S}(\varepsilon) \subset \mathcal{S}_{*}(0) \subset \underline{\lim }_{\varepsilon \searrow 0} \mathcal{S}(\varepsilon) .
$$

Now, the proof is divided into two subsections, which are concerned with respective inclusions as in (22).

5.1. Proof of $\overline{\lim }_{\varepsilon \backslash 0} \mathcal{S}(\varepsilon) \subset \mathcal{S}_{*}(0)$. This inclusion is proved by means of a contradiction argument. So, let us assume that $w_{*} \in \varlimsup_{\lim _{\varepsilon}{ }_{0}} \mathcal{S}(\varepsilon) \backslash \mathcal{S}_{*}(0)$. Then, by the definition of the 
limit-supremum, we can take sequences $\left\{\varepsilon_{i}\right\} \subset\left(0, \varepsilon_{\kappa}\right)$ and $\left\{w_{i}\right\} \subset D_{*}$, such that:

$$
\left\{\begin{array}{l}
\bullet w_{i} \in \mathcal{S}\left(\varepsilon_{i}\right) \backslash \mathcal{S}_{*}(0)=\bigcup_{n=1}^{n(\varepsilon)} \mathcal{S}_{n}\left(\varepsilon_{i}\right), i=1,2,3, \ldots \\
\bullet \varepsilon_{i} \searrow 0, \quad w_{i} \rightarrow w_{*} \text { in } L^{2}(\Omega), \text { and } w_{i}(x) \rightarrow w_{*}(x) \text { a.e. } \\
x \in \Omega, \text { as } i \rightarrow+\infty .
\end{array}\right.
$$

Additionally, since $n(\varepsilon) \leq n(0)$ for $0<\varepsilon<\varepsilon_{\kappa}$, we may say that $w_{i} \in \mathcal{S}_{n}\left(\varepsilon_{i}\right), i=$ $1,2,3, \ldots$, for a certain number $1 \leq n \leq n(0)$, by taking a subsequence if necessary. Therefore, on account of conditions $(\mathrm{s} 0)_{\varepsilon^{-}}(\mathrm{s} 3)_{\varepsilon}$ as in Theorem 1 and conditions $(\mathrm{s} 0)_{0^{-}}$ $(\mathrm{s} 2)_{0}$ as in Proposition 2, it is not difficult to verify that $w_{*} \in \mathcal{S}_{n}(0)$. Also, from the convergence as in (23), we further see that $x_{k}^{L}=x_{k}^{R}$ for $k=1, \ldots, n$.

Hence, as the last possibility, the solution $w_{*} \in \mathcal{S}_{n}(0)$ is supposed to satisfy that:

$$
\left|c_{k}\right|=1 \text { and }\left|c_{k}+\theta_{*}\right| \mathcal{L}^{1}\left(J_{k}\right)=2 \kappa \text { for all } k=0,1, \ldots, n \text {. }
$$

But even in this case, the following contradictory inequality:

$$
\mathcal{L}^{1}(\Omega)=\sum_{k=0}^{n} \mathcal{L}^{1}\left(J_{\varepsilon_{i}, k} \cap \Omega\right)>\sum_{k=0}^{n} \mathcal{L}^{1}\left(J_{k} \cap \Omega\right)=\mathcal{L}^{1}(\Omega), i=1,2,3, \ldots,
$$

will be seen from (III) of Proposition 1.

5.2. Proof of $\mathcal{S}_{*}(0) \subset \underline{\lim }_{\varepsilon \backslash 0} \mathcal{S}(\varepsilon)$. Let us take any $w_{*} \in \mathcal{S}_{*}(0)$. Then, in the light of the definition of the limit-infimum, all we have to do will be to construct a sequence $\left\{w_{\varepsilon} \mid 0<\varepsilon<\varepsilon_{\kappa}\right\} \subset D_{*}$ of approximating solutions $w_{\varepsilon}$, such that:

$$
w_{\varepsilon} \in \mathcal{S}(\varepsilon) \text { for all } 0<\varepsilon<\varepsilon_{\kappa} \text {, and } w_{\varepsilon} \rightarrow w_{*} \text { in } L^{2}(\Omega) \text { as } \varepsilon \searrow 0 \text {. }
$$

The construction is as follows.

First, let us consider $w_{*}$ constant. Under this situation, we immediately find the approximating sequence by setting $w_{\varepsilon} \equiv w_{*}$ in $\Omega$ for all $0<\varepsilon<\varepsilon_{\kappa}$, since:

$$
\mathcal{S}_{0}(\varepsilon)=\mathcal{S}_{0}(0)=\mathcal{S}_{*, 0}(0)=\left\{-1,-\theta_{*}, 1\right\} \text { for all } 0<\varepsilon<\varepsilon_{\kappa} .
$$

Secondly, let $w_{*} \in \mathcal{S}_{*, n}(0)$ for some $1 \leq n \leq n(0)$, and let us take the index $1 \leq k_{0} \leq n$ as in (13).

Under this situation, we can construct the required approximating sequence $\left\{w_{\varepsilon} \mid 0<\right.$ $\left.\varepsilon<\varepsilon_{\kappa}\right\}$ by the continuous dependence of the constant $\mu_{\varepsilon}^{(a)}$, mentioned in (v) of (III) of Proposition 1 . Here, we pick up the following two cases of $w_{*}$, to show the essence of the construction methods.

Case $A: n=1, c_{k}=(-1)^{k}$ for $k=0,1$, and $\left|c_{1}+\theta_{*}\right| \mathcal{L}^{1}\left(J_{1}\right)>2 \kappa\left(k_{0}=1\right)$. In this case, we find, for example, the following functions $w_{\varepsilon}\left(0<\varepsilon<\varepsilon_{\kappa}\right)$, given by: $w_{\varepsilon}(x):=\left\{\begin{aligned}\left(\gamma_{\varepsilon}^{\left(c_{0}\right)}\left(x ; \ell_{A, \varepsilon}\right)+\theta_{*}\right) \chi_{\left(0, \ell_{A, \varepsilon}\right)}(x) \\ \quad+\left(\gamma_{\varepsilon}^{\left(c_{1}\right)}\left(x-1 ; 1-\ell_{A, \varepsilon}\right)+\theta_{*}\right) \chi_{\left(\ell_{A, \varepsilon}, 1\right)}(x)-\theta_{*}, \text { if } 0<\varepsilon<\varepsilon_{A}, \\ -\theta_{*}, \text { if } \varepsilon_{A} \leq \varepsilon<\varepsilon_{\kappa}, \text { for any } x \in \Omega,\end{aligned}\right.$

as members of the approximating sequence, where $\gamma_{\varepsilon}^{(\cdot)}(\cdot ; \cdot)$ is the $C^{1}$-function given in (10), $0<\varepsilon_{A}<\varepsilon_{\kappa}$ is a small constant taken to satisfy $\max \left\{x_{1}^{L}, \mu_{\varepsilon_{A}}^{(1)}\right\}+\mu_{\varepsilon_{A}}^{(-1)}<1\left(=\mathcal{L}^{1}(\Omega)\right)$, and for any $0<\varepsilon<\varepsilon_{A}, \ell_{A, \varepsilon}$ is a positive constant given by $\ell_{A, \varepsilon}:=\max \left\{x_{1}^{L}, \mu_{\varepsilon}^{\left(c_{0}\right)}\right\}$. In 
fact, by using the properties as in (III) of Proposition 1, it is not difficult to check that this sequence $\left\{w_{\varepsilon} \mid 0<\varepsilon<\varepsilon_{\kappa}\right\}$ fulfills the approximating property as in (24).

Case $B: n=1,-\theta_{*}<c_{0}<1$ and $\left|c_{1}+\theta_{*}\right| \mathcal{L}^{1}\left(J_{1}\right)=2 \kappa\left(k_{0}=0\right)$. In this case, the constituent functions $w_{\varepsilon}\left(0<\varepsilon<\varepsilon_{\kappa}\right)$ are, for example, given as:

$w_{\varepsilon}(x):=\left\{\begin{array}{c}\left(\gamma_{\varepsilon}^{\left(c_{B, \varepsilon}\right)}\left(x ; 1-\mu_{\varepsilon}^{\left(c_{1}\right)}\right)+\theta_{*}\right) \chi_{\left(0,1-\mu_{\varepsilon}^{\left(c_{1}\right)}\right)}(x) \\ \quad+\left(\gamma_{\varepsilon}^{\left(c_{1}\right)}\left(x-1 ; \mu_{\varepsilon}^{\left(c_{1}\right)}\right)+\theta_{*}\right) \chi_{\left(1-\mu_{\varepsilon}^{\left(c_{1}\right)}, 1\right)}(x)-\theta_{*}, \text { if } 0<\varepsilon<\varepsilon_{B}, \\ -\theta_{*}, \text { if } \varepsilon_{B} \leq \varepsilon<\varepsilon_{\kappa}, \quad \text { for any } x \in \Omega .\end{array}\right.$

Here, $0<\varepsilon_{B}<\varepsilon_{\kappa}$ is a small constant taken to satisfy $\mu_{\varepsilon_{B}}^{(1)}+\mu_{\varepsilon_{B}}^{\left(c_{1}\right)}<1$, and for any $0<\varepsilon<\varepsilon_{B}, c_{0}<c_{B, \varepsilon}<1$ is a constant taken to satisfy $\mu_{\varepsilon}^{\left(c_{B}, \varepsilon\right)}+\mu_{\varepsilon}^{\left(c_{1}\right)}=1$. Then, from (v) of (III) of Proposition 1, it will be seen that the constants $\varepsilon_{B}$ and $c_{B, \varepsilon}\left(0<\varepsilon<\varepsilon_{B}\right)$ are well-defined, and moreover, the sequence $\left\{w_{\varepsilon} \mid 0<\varepsilon<\varepsilon_{\kappa}\right\}$ fulfills (24).

In other cases, we will find the approximating sequence $\left\{w_{\varepsilon} \mid 0<\varepsilon<\varepsilon_{\kappa}\right\}$ analogously.

Acknowledgements. This work is partially supported by the Grant-in-Aid for Scientific Research, No. 17740099.

\section{References}

[1] L. Ambrosio, N. Fusco and D. Pallara, Functions of Bounded Variation and Free Discontinuity Problems, Oxford Science Publications, 2000.

[2] J.-P. Aubin and H. Frankowska, Set-Valued Analysis, Systems and Control: Foundations and Applications 2, Birkhäuser, Boston, 1990.

[3] N. Kenmochi and K. Shirakawa, A variational inequality for total variation functional with constraint, Nonlinear Anal. 46 (2001), 435-455.

[4] U. Mosco, Convergence of convex sets and of solutions of variational inequalities, Advances in Math. 3 (1969), 510-585.

[5] K. Shirakawa, Asymptotic convergence of $p$-Laplace equations with constraint as $p$ tends to 1, Math. Methods Appl. Sci. 25 (2002), 771-793.

[6] K. Shirakawa and M. Kimura, Stability analysis for Allen-Cahn type equation associated with the total variation energy, Nonlinear Anal. 60 (2005), 257-282.

[7] A. Visintin, Models of Phase Transitions, Progress in Nonlinear Differential Equations and Their Applications 28, Birkhäuser, Boston, 1996. 